\title{
Differential $G$ protein subunit expression by prostate cancer cells and their interaction with CXCR5
}

Christelle P El-Haibi ${ }^{1}$, Praveen Sharma ${ }^{2}$, Rajesh Singh ${ }^{3}$, Pranav Gupta ${ }^{3}$, Dennis D Taub ${ }^{4}$, Shailesh Singh $^{3}$ and James W Lillard, $\mathrm{Jr}^{3^{*}}$

\begin{abstract}
Background: Prostate cancer (PCa) cell lines and tissues differentially express CXCR5, which positively correlate with PCa progression, and mediate PCa cell migration and invasion following interaction with CXCL13. However, the differential expression of $\mathrm{G}$ protein $\mathrm{a}, \beta$, and $\gamma$ subunits by PCa cell lines and the precise combination of these proteins with CXCR5 has not been elucidated.

Methods: We examined differences in G protein expression of normal prostate (RWPE-1) and PCa cell lines (LNCaP, C4-2B, and PC3) by western blot analysis. Further, we immunoprecipitated CXCR5 with different $G$ protein subunits, and CXCR4, following CXCL13 stimulation. To investigate constitutive coupling of CXCR5 with CXCR4 and PAR-1 we performed invasion assay in PCa cells transfected with $G_{a q / i 2}$ or $G_{a 13}$ siRNA, following CXCL13 treatment. We also investigated Rac and RhoA activity by G-LISA activation assay in PCa cells following CXCL13/thrombin stimulation.

Result: Of the $22 \mathrm{G}$ proteins studied, $G_{a i 1-3,}, G_{\beta 1-4}, G_{\gamma 5}, G_{\gamma 7}$, and $G_{\gamma 10}$ were expressed by both normal and PCa cell lines. $G_{\text {as }}$ was moderately expressed in C4-2B and PC3 cell lines, $G_{a q / 11}$ was only present in RWPE-1 and LNCaP cell lines, while $G_{a 12}$ and $G_{a 13}$ were expressed in C4-2B and $P C 3$ cell lines. $G_{\gamma 9}$ was expressed only in PCa cell lines. $G_{\alpha 16,} G_{\beta 5}, G_{Y 1-4}$, and $G_{Y 13}$ were not detected in any of the cell lines studied. Surprisingly, CXCR4 co-immunoprecipitated with CXCR5 in PCa cell lines irrespective of CXCL13 treatment. We also identified specific $\mathrm{G}$ protein isoforms coupled to CXCR5 in its resting and active states. $G_{a q / 11}, G_{\beta 3}, G_{\gamma 9}$ in $L N C a P$ and $G_{a i 2} G_{\beta 3}, G_{\gamma 9}$ in $C 4-2 B$ and PC3 cell lines, were coupled to CXCR5 and disassociated following CXCL13 stimulation. Interestingly, $G_{a 13}$ Co-immunoprecipitated with CXCR5 in CXCL13-treated, but not in untreated PCa cell lines. Inhibition of $G_{a q / 12}$ significantly decreased the ability of cells to invade, whereas silencing $G_{a 13}$ did not affect CXCL13-dependent cell invasion. Finally, CXCL13 treatment significantly increased Rac activity in $G_{a q / 12}$ dependent manner, but not RhoA activity, in PCa cell lines.

Conclusions: These findings offer insight into molecular mechanisms of PCa progression and can help to design some therapeutic strategies involving CXCR5 and/or CXCL13 blockade and specific G protein inhibition to abrogate PCa metastasis.
\end{abstract}

Keywords: Prostate cancer, Chemokine, G proteins, G protein-coupled receptor

\footnotetext{
* Correspondence: jlillard@msm.edu

${ }^{3}$ Department of Microbiology, Biochemistry, \& Immunology, Morehouse

School of Medicine, Atlanta, GA, USA

Full list of author information is available at the end of the article
}

\section{Biomed Central}

(c) 2013 El-Haibi et al.; licensee BioMed Central Ltd. This is an Open Access article distributed under the terms of the Creative Commons Attribution License (http://creativecommons.org/licenses/by/2.0), which permits unrestricted use, distribution, and reproduction in any medium, provided the original work is properly cited. 


\section{Background}

G protein-coupled receptors (GPCRs) are divided into three broad classes based on the similarity of the transmembrane sequences and the nature of their ligand [1]. Chemokine receptors are categorized under the superfamily of Class A Rhodopsin-like GPCRs [2]. GPCRs interact with heterotrimeric guanine nucleotide-binding proteins (G proteins) composed of $\alpha, \beta$, and $\gamma$ subunits present on the inner surface of the plasma membrane. After ligand binding, the receptor elicits a conformational alteration resulting in the exchange of guanosine diphosphate (GDP) for guanosine triphosphate (GTP) by the $G_{\alpha}$ subunit. This leads to heterotrimer dissociation and stimulation of downstream effector molecules to initiate intracellular signaling cascades $[1,3,4] . G_{\alpha}$ subunits are divided into four families $G_{\alpha \mathrm{\alpha}}, G_{\alpha i}, G_{\alpha q / 11}$, and $G_{\alpha 12 / 13}$ based on sequence homology and functional similarities. $\mathrm{G}_{\alpha \mathrm{s}}$ proteins are known to stimulate adenylyl cyclases $(A C)$, while $G_{\alpha i}$ proteins inhibit AC and activate phosphodiesterases. Alternatively, $G_{\alpha q / 11}$ proteins regulate the activity of phosphatidylinositol-specific phospholipases to generate lipid second messengers, and $G_{\alpha 12 / 13}$ proteins regulate the small guanine triphosphate (GTPases). On the other hand, $G$ protein $\beta$ and $\gamma$ subunits function as a tightly associated complex to modulate the activity of several effectors including $\mathrm{AC}$, protein tyrosine kinases (e.g., Src family tyrosine kinases), phosphoinositide-3 kinase (PI3K) $\gamma$, GPCR kinases (GRKs), and $\mathrm{Ca}^{+2}$ as well as $\mathrm{K}^{+}$ion channels $[4,5]$.

$\mathrm{G}_{\alpha}$ subunits are encoded by 17 genes (Gnas, Gnasxl, Gnal, Gnai1, Gnai2, Gnai3, Gnao, Gnaz, Gnag, Gnat1, Gnat2, Gnaq, Gna11, Gna14, Gna15, Gna12, and Gna13). There are five known genes encoding $G_{\beta}$ subunits (Gnb1, Gnb2, Gnb3, Gnb4, and Gnb5) and 12 genes encoding $\mathrm{G}_{\gamma}$ subunits (Gngt1, Gngt2, Gng2, Gng3, Gng4, Gng5, Gng7, Gng8, Gng10, Gng11, Gng12, and Gng13) [3,6,7]. A large number of potential combinations of $G_{\alpha / \beta / \gamma}$ heterotrimers can form; however, not all associations are functional and they vary in their affinity for distinct GPCRs $[8,9]$. G proteins also exhibit tissuespecific expression. Most G proteins are ubiquitously present in several tissues, but a smaller subset is confined to specialized cell types $[7,10]$.

Several studies have reported the role of G proteins in different human diseases [11]. Comparatively, less is known regarding the expression of these signaling proteins by PCa cells. PCa cells express a repertoire of chemokine receptors that contribute to disease progression and metastasis $[12,13]$. In this regard, we have shown that PCa cell lines differentially express CXCR5, and this expression positively correlates with the ability of cell lines to migrate and invade extracellular matrix components following interaction with CXCL13 [14,15]. To our knowledge, neither the differential expression of $\mathrm{G}$ protein $\alpha, \beta$, and $\gamma$ subunits by PCa cell lines nor specific $\mathrm{G}$ protein interactions with CXCR5 have been described. Here, we elucidate the differences in G protein isoforms expressed by normal and tumorigenic prostate cell lines. We also identified the specific $G$ protein isoforms coupled to CXCR5 in the presence or absence of CXCL13 stimulation.

\section{Results}

Endogenous expression of $G_{\alpha}$ protein subunits by PCa cells In light of the diversity of $G$ protein isoforms and functions, we revealed the differential expression of $G$ protein $\alpha, \beta$, and $\gamma$ isoforms by Western blot analysis of total lysates of untreated PCa and normal cell lines. Our results show that $\mathrm{G}_{\mathrm{\alpha i}}$ subunit (isoforms 1,2 , and 3) are widely expressed by RWPE-1, LNCaP, C4-2B, and PC3 cell lines (Figure 1A). The $G_{\alpha s}$ subunit was expressed by all cell lines studied, with reduced levels in C4-2B and PC3 cell lines. $G_{\alpha 12}$ was expressed by hormone refractory cell lines C4-2B and PC3, but was absent in RWPE-1 and LNCaP cell lines. $G_{\alpha 13}$ was unequally distributed among the four cell lines examined, showing elevated levels in C4-2B and PC3 cell lines (Figure 1A). The $G_{\alpha q / 11}$ subunit was confined to the androgen-dependent cell lines - RWPE-1 and $\mathrm{LNCaP}$ and not detected in hormone refractory cell lines, C4-2B and PC3 (Figure 1A). Lastly, $\mathrm{G}_{\alpha 16}$ was not detected in any of the tested cell lines (data not shown), probably due to its specificity for hematopoietic cells [16].

\section{Endogenous expression of $\mathrm{G}_{\beta \gamma}$-protein subunits by prostate cells}

Except for the $G_{\beta 5}$ isoform (data not shown), which reported to be largely expressed by brain tissue [5,9], all other $G_{\beta}$ isoforms were present in all prostate cell lines examined (Figure 1B). The expression of $G_{\gamma}$ subunits exhibited a distinctive pattern where only isoforms $[5,7,9,10]$ were detected in the cell lines studied. As expected, $G_{\gamma 1-4}$ and $G_{\gamma 13}$ were not detected in any of the cell lines tested (data not shown) (Figure 1C), because they have previously shown to be confined to retinal rods, brain tissue, and taste buds respectively [3]. Interestingly, $G_{\gamma 9}$ was expressed at very low levels in the normal prostate cell line, but was significantly expressed in all of the PCa cell lines tested.

\section{Specific G proteins coupled to CXCR5 in PCa cell lines}

It is now well established that chemokine receptors are often up-regulated and potentially influence the tumor behavior in a variety of human cancers including prostate cancer. Here, we demonstrate that CXCR5 is highly expressed by PCa cell lines (LNCaP, C4-2B, and PC3), but in low to undetectable amount by the normal prostate cell line, RWPE-1 (Figure 2A). Chemokine receptors are usually, but not exclusively, coupled to $G_{\alpha i}$ subclass 

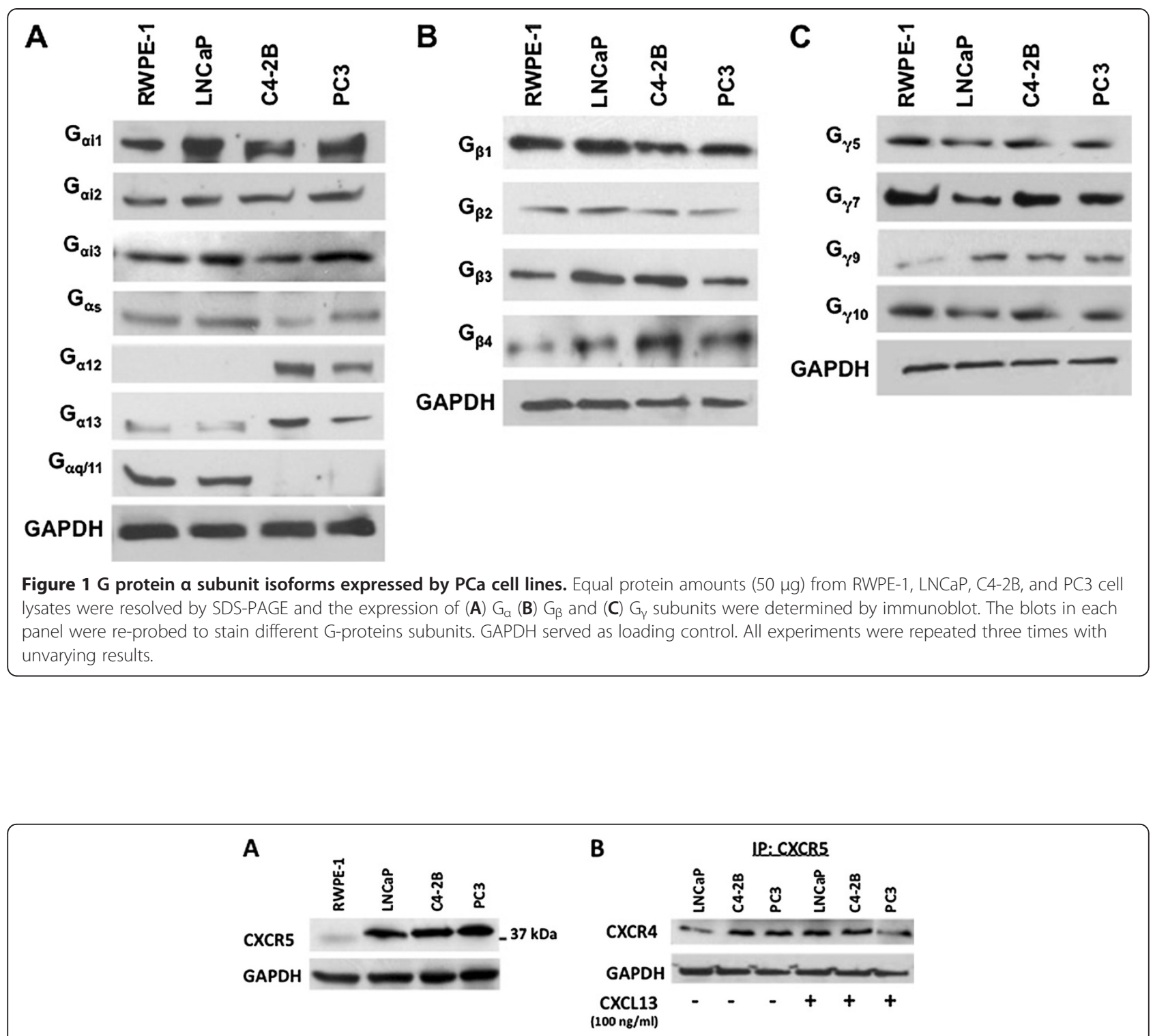

C

D $\quad$ R: $:$ CXCR5.
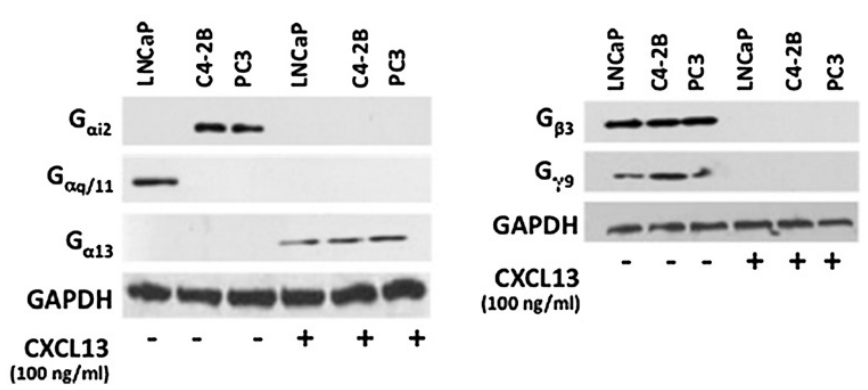

Figure 2 Expression of CXCR4, CXCR5 and associated G proteins in PCa cell lines. (A) Equal protein amounts (50 $\mu \mathrm{g})$ from RWPE-1, LNCaP, C4-2B, and PC3 cell lysates were resolved by SDS-PAGE and the expression of CXCR5 (37 kDa) was determined by immunoblot. (B) Western blot analysis of CXCR4 expression with and without CXCL13 treatment. (C) \& (D) Cell lines were treated with or without CXCL13 and lysed. CXCR5 was immunoprecipitated (IP) to pull down associated proteins from total cell lysates. The IP cell lysates were resolved by SDS-PAGE and the expression of (C) $G_{a i 1}, G_{a i 2}, G_{a i 3}, G_{a 1}, G_{a q} / 11, G_{a 12}, G_{a 13}$ (D) $G_{\beta 1}, G_{\beta 2}, G_{\beta 3}, G_{\beta 4}$, and $G_{\gamma 5}, G_{\gamma 7}, G_{\gamma 9}, G_{\gamma 10}$ were examined by immunoblot. The blots in panel $C$ and $D$ were stripped each time and re-probed to stain different $G_{\alpha}, G_{\beta}$, and $G_{\gamma}$ protein subunits. In all the experiments, GAPDH served as loading control. All experiments were repeated three times with unvarying results. 
of $G$ proteins [17]. In this study, we demonstrate that only $\mathrm{G}_{\text {ai2 }}$ co-immunoprecipitated with CXCR5 in untreated C4-2B and PC3 cell lines in the absence of agonist, while $\mathrm{G}_{\alpha \mathrm{q} / 11}$ associates with CXCR5 in untreated LNCaP cells. $\mathrm{G}_{\alpha 13}$ co-immunoprecipitated with CXCR5 in all three PCa cell lines treated with CXCL13, but was not detected in untreated cells (Figure $2 C$ ). $G_{\beta 3}$ and $G_{\gamma 9}$ co-immunoprecipitated with CXCR5 in the absence of CXCL13 in all PCa cell lines used (Figure 2D). This $\mathrm{G}_{\beta 3 / \gamma 9}$ complex was not detected following CXCL13 stimulation indicating its ligand-induced dissociation from the receptor. The other $G_{\alpha(i 1, i 3),} G_{s}, G_{\alpha 12}, G_{\beta}(1,2,4)$ and $G_{\gamma}(5,7,10)$ subunits which were detected in PCa cell lines (Figure 1B and $1 C$ ) were not co-immunoprecipitated with CXCR5 in presence or absence of agonist (data not shown).

Validation and significance of $\mathrm{G}_{\mathrm{aq} / 11 /} \mathrm{G}_{\beta 3} / \mathrm{G}_{\gamma 9}$ and $\mathrm{G}_{\mathrm{ai} 2 /} \mathrm{G}_{\beta 3 /}$ $\mathrm{G}_{\gamma 9}$ binding to CXCR5 in LNCaP, and C4-2B, and PC3 cell lines respectively

To further validate differences observed in $G_{\alpha}$ subunit(s) coupling and uncoupling to CXCR5 in CXCL13-treated versus untreated cells, we separately immunoprecipitated $\mathrm{G}_{\mathrm{\alpha q} / 11}$ and $\mathrm{G}_{\mathrm{\alpha i} 2}$ subunits in untreated and CXCL13-treated PCa cells and immunoblotted for CXCR5. Our results provide the first evidence of multifunctional coupling of CXCR5 to different types of $G$ proteins favoring a pertussis toxin-insensitive signaling pathway mediated by $G_{\alpha q / 11}$ in LNCaP cells and a pertussis toxin-sensitive signaling pathway mediated by $\mathrm{G}_{\alpha \mathrm{i} 2}$ in C4-2B and PC3 cells (Figure 3).

\section{Association of $\mathrm{G}_{\mathrm{a} 13}$ protein, CXCR4, and PAR-1 with} CXCR5 in CXCL13-treated PCa cell lines

One surprising result was the association of the $G_{\alpha 13}$ subunit with CXCR5 in PCa cell lines treated with CXCL13, but not in untreated cells. Thus, it was critical to confirm this finding by immunoprecipitating $G_{\alpha 13}$ protein from CXCL13-treated and untreated PCa cells, and immunoblotting for CXCR5. Results confirm that coupling of $\mathrm{G}_{\alpha 13}$ to CXCR5 is specific to CXCL13treated cells (Figure $3 \mathrm{C}$ ). It has been reported that proteinase activated receptor-1 (PAR-1) is capable of bypassing signaling through $\mathrm{G}_{\alpha \mathrm{i}}$-pathway to support $\mathrm{G}_{\alpha 12 / 13}$-dependent mechanisms, enhancing cellular proliferation, invasion, and metastasis [18]. We therefore examined the association of PAR-1 with $G_{\alpha 13}$ and showed that CXCR5 and PAR-1 are linked to $G_{\alpha 13}$ following treatment with CXCL13 (Figure 4A).

The presence of CXCR4 in CXCR5 immunoprecipitants (with or without CXCL13 treatment) offers the first evidence of CXCR5 association with CXCR4 (Figure 2B). These interactions could potentially support CXCR4CXCR5 signaling crosstalk. Moreover, the ability of
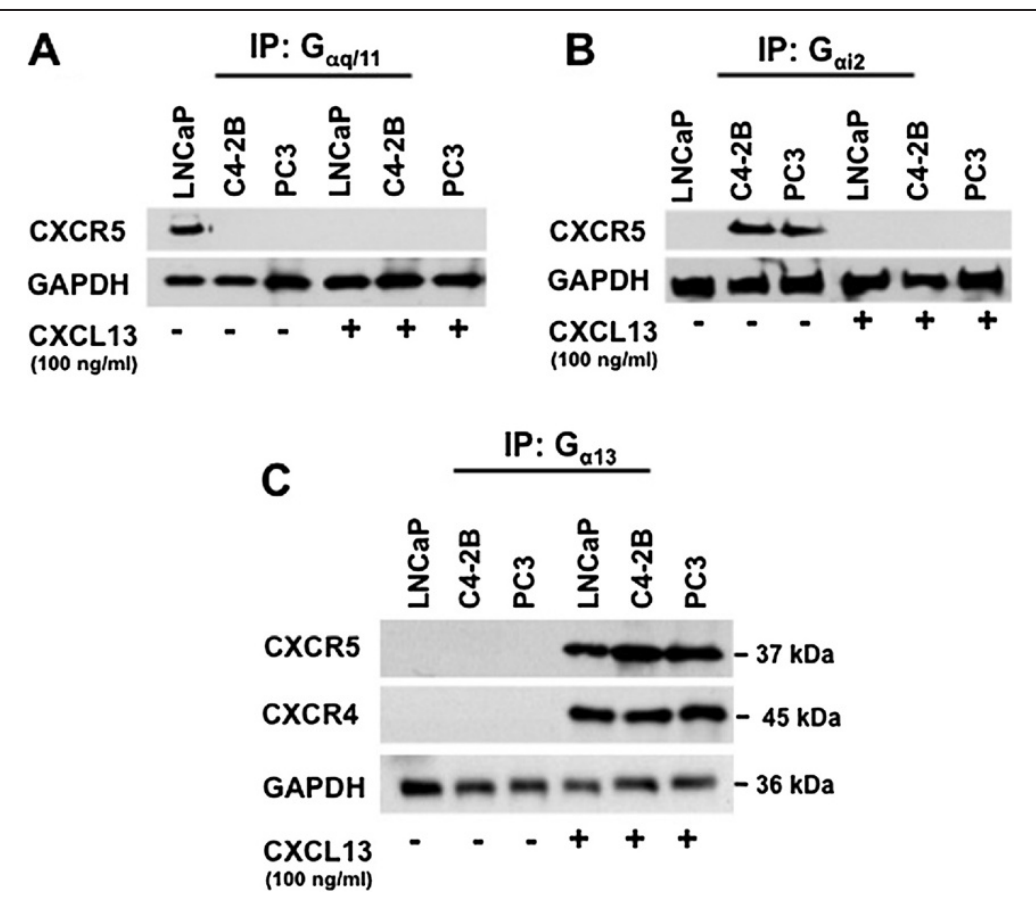

Figure 3 Validation of $\mathrm{G}_{\mathrm{aq} / 11}, \mathrm{G}_{\mathrm{ai2}}$, and $\mathrm{G}_{\mathrm{a} 13}$ protein association with CXCR5. Cell lines were treated with or without CXCL13 and lysed. (A) $G_{\text {aq } / 11}$ and (B) $G_{\text {ai2 }}$ were immunoprecipitated (IP) from total cell lysates. The IP cell lysates were resolved by SDS-PAGE and CXCR5 expression was examined by immunoblot. (C) Identification of CXCR4 and CXCR5 coupled to $G_{a 13}$ following CXCL13 stimulation. Cell lines were treated with or without CXCL13 and lysed. Antibody against $G_{a 13}$ was used to immunoprecipitate (IP) it from total cell lysates. The IP cell lysates were resolved by SDS PAGE and immunoblotted for CXCR5 followed by CXCR4, after stripping. In all the experiments, GAPDH served as loading control. All experiments were repeated three times with unvarying results. 
A

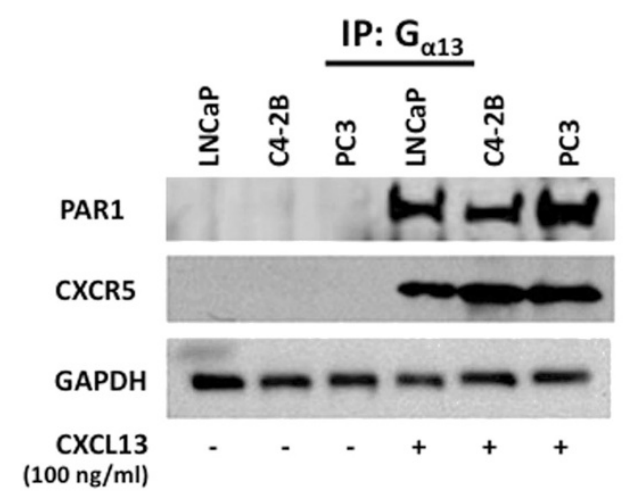

B

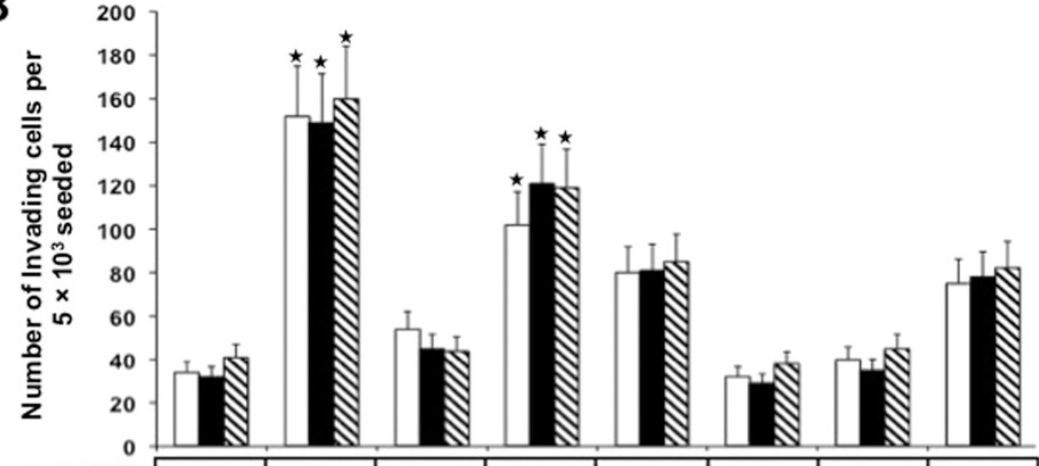

Control siRNA

Gaq/i2 siRNA

Ga13 siRNA

CXCL13

Thrombin

\begin{tabular}{|c|c|c|c|c|c|c|c|}
\hline+ & + & - & - & + & + & - & - \\
\hline- & - & + & - & - & - & + & - \\
\hline- & - & - & + & - & - & - & + \\
\hline- & + & + & + & + & - & + & + \\
\hline- & - & - & - & + & + & + & + \\
\hline
\end{tabular}

C (i) $\mathrm{G}_{\alpha \mathrm{i}}$ and $\mathrm{G}_{\mathrm{q} / 11}$

Lamellipodia (Rac)

\begin{tabular}{l|l} 
& Rac1 \\
\hline NA & $0 \%$ \\
CXCL13 + control siRNA & $395 \% *$ \\
Thrombin + control siRNA & $26 \%$ \\
CXCL13 $\rightarrow$ Thrombin & $265 \% *$ \\
$\mathrm{G}_{\alpha \mathrm{q} / 12}$ SiRNA + CXCL13 & $-17 \%$ \\
$\mathrm{G}_{\alpha 13}$ SiRNA + CXCL13 & $171 \% *$
\end{tabular}

$\downarrow$

Cell invasion

"CXCL13/CXCR5" (ii)

$\mathrm{G}_{12 / 13}$

$\downarrow$

Stress Fibers (Rho)

\begin{tabular}{l|l} 
& RhoA \\
\hline NA & $0 \%$ \\
CXCL13 + control siRNA & $6 \%$ \\
Thrombin + control siRNA & $483 \% *$ \\
CXCL13 $\rightarrow$ Thrombin & $8 \%$ \\
$\mathrm{G}_{\alpha \mathrm{q} / 12}$ SiRNA + Thrombin & $216 \% *$ \\
$\mathrm{G}_{\alpha 13}$ SiRNA + Thrombin & $11 \%$
\end{tabular}

$\downarrow$

Cell adhesion

Thrombin/PAR-1

Figure 4 (See legend on next page.) 
(See figure on previous page.)

Figure $4 \mathrm{G}_{\mathrm{a} 13}$ association with PAR-1 and CXCR5, and $\mathrm{G}_{\mathrm{a} 13}$ and $\mathrm{G}_{\mathrm{ai2}}$ contribution to PCa cell lines invasion and Rac/Rho activation. (A) Cell lines were treated with or without CXCL13 and lysed. Antibody against $\mathrm{G}_{\mathrm{a} 13}$ was used to immunoprecipitate (IP) it from total cell lysates. The IP cell lysates were resolved by SDS PAGE and immunoblotted for PAR-1 followed by CXCR5, after stripping. GAPDH served as a loading control. (B) Invasion of LNCaP, C4-2B and PC3 cells was assessed using BD Matrige ${ }^{\mathrm{TM}}$ invasion chamber. The assay was performed using LNCaP (open bars), C4-2B (solid bars) and PC3 (hashed bars) cells transfected and/or treated with control siRNA, Gaq/ai2 siRNA, or Ga13 siRNA duplex, and CXCL13 and/or Thrombin for $8 \mathrm{~h}$ and the cells that migrated to the lower surface of the membrane were counted by microscopy at $40 \mathrm{X}$ magnification. CXCL13-treated cells exhibited an enhanced ability to invade Matrigel. Abrogation of $\mathrm{G}_{\mathrm{aq} / \mathrm{i2}}$ decreased the ability of cells to invade whereas silencing of $\mathrm{G}_{\mathrm{a} 13}$ did not affect cell invasion. (C) Rac and RhoA protein expression were determined in CXCL13 and/or thrombin treated LNCaP, C4-2B, and PC3 cells. (i) shows differential expression of Rac protein, involved in lamellipodia formation, in response to CXCL13, thrombin, CXCL13 followed by thrombin and from cells transfected with $\mathrm{G}_{\mathrm{aq} / \mathrm{i} 2 / 13}$ siRNA in different experiments. (ii) shows differential expression of RhoA protein, involved in stress fiber formation and cell adhesion, in response to CXCL13, thrombin, CXCL13 followed by thrombin and from cells transfected with $\mathrm{G}_{\mathrm{a} 13}$ siRNA in different experiments. All experiments were repeated at least three times and results were in accordance with each other.

CXCR4 to engage in $G_{\alpha 13}$-mediated cell signaling events that activate Rho pathways leading to cell adhesion has been previously demonstrated [19]. $G_{\alpha 13}$ association with CXCR5, CXCR4 and PAR-1 after CXCL13 treatment (Figures 3C \& 4A) alludes to chemokine receptor oligomer formation or the recruitment of other GPCR- $G_{\alpha 13}$ associated signaling complexes after stimulation, which could presumably potentiate synergistic or additional biological events, respectively [20,21].

It is plausible that the CXCL13:CXCR5 axis regulates cell migration by desensitizing CXCR4 and conditional coupling of CXCR5 with PAR-1. Therefore, constitutive coupling of CXCR5 with CXCR4 and PAR-1 after CXCL13 ligation in PCa cells could be another mechanism through which CXCL13 sequesters factors hampering cell migration. To investigate whether this hypothesis holds true, we allowed LNCaP, C4-2B, and PC3 cells previously transfected with $G_{\alpha q / i 2}$ or $G_{\alpha 13}$ siRNA duplexes to invade across a Matrigel membrane following treatment with CXCL13 or thrombin, which are activating ligands of CXCR5 and PAR-1, respectively. Control siRNA duplex-treated PCa cells exhibited increased invasive potential to CXCL13 (Figure 4B). While abrogation of $\mathrm{G}_{\alpha \mathrm{q} / \mathrm{i} 2}$ significantly decreased the ability of cells to invade, silencing $G_{\alpha 13}$ did not affect CXCL13dependent cell invasion. In contrast, PCa cell lines did not invade in response to thrombin alone, but were moderately invasive in the presence of CXCL13 and thrombin. This invasive potential was also $G_{\alpha q / i 2}$-dependent, but $G_{\alpha 13}$ independent. Taken together, these observations suggest CXCL13 is signaling independently of the PAR- $1 / G_{\alpha 13}$ complex and mainly through $\mathrm{CXCR} 5 / \mathrm{G}_{\alpha \mathrm{\alpha q} / \mathrm{i} 2}$ to promote PCa cell invasion.

\section{CXCL13, Thrombin, $\mathrm{G}_{\mathrm{aq} / \mathrm{i} 2}$ protein, and $\mathrm{G}_{\mathrm{a} 13}$ protein mediated Rac and RhoA activation in PCa cell lines} $G$ proteins have been shown to differentially activate three members of the Rho family of GTPases (Rac, Cdc42, and RhoA). Our data show that $G_{\alpha q / 11 / \beta 3 / \gamma 9}$ and $\mathrm{G}_{\alpha \mathrm{i} 2 / \beta 3 / \gamma 9}$ proteins dissociated from CXCR5 after
CXCL13 stimulation. This uncoupling is thought to be the result of $G$ protein subunit activation, which stimulates downstream effector molecules, including RhoA and Rac. We therefore performed Rac and RhoA activity assays on CXCL13 and thrombin-treated PCa cells. CXCL13 treatment resulted in a 395\% increase in Rac activity, but no change in RhoA activity (Figure 4C). Correspondingly, thrombin-treated PCa cells displayed no significant increase in Rac activity. CXCL13-mediated Rac activation was $G_{\alpha q / i 2}$-dependent, while thrombininduced RhoA activation was $G_{\alpha 13}$-dependent and $G_{\alpha q / i 2}$ independent. Interestingly, treatment of cells with CXCL13, 5 min before thrombin stimulation did not significantly effect Rac activation, but abrogated thrombindependent RhoA activation. Together, our results show CXCL13 stimulation biases PCa cells to invade or migrate, instead of adhere, even in the presence of a potent adherence signal, i.e., thrombin-PAR-1 interactions.

\section{Discussion}

GPCR mediated heterotrimeric $G$ protein signaling is known to regulate cellular motility, growth and differentiation, and gene transcription, three factors central to the biology of cancer. Depending on the physiologic function, expression of $\mathrm{G}$ protein(s) subunit isoforms may vary from one cell type to other. $G_{\alpha i}$ subunit inhibits the production of cAMP from ATP. In our study, we found constitutive expression of $G_{\alpha i}$ subunit isoforms in all the cell lines tested. This is in tune with the earlier reports stating that $G_{\alpha i}$ subunit isoforms are the most ubiquitously expressed $G$ protein $\alpha$ isoforms $[7,10]$. Moreover, studies of tissue samples obtained from patients with T2 stage PCa revealed low levels of $G_{\alpha s}$ subunit compared to high levels in normal controls [22]. $G_{\alpha 12}$ and $G_{\alpha 13}$ levels were significantly elevated by PC3 and DU-145 cell lines, than compared to PrEC and LNCaP cell lines [23,24]. We found similar results, where $G_{\alpha 12}$ was detected only in hormone refractory C4-2B and PC3 cell lines, whereas $G_{\alpha 13}$ was significantly elevated in these cell lines. $G_{\beta 1-4}$ and $G_{\gamma 5,7,9,10}$ were 
expressed in all the cell lines tested. If all of these $G_{\beta 1-4}$ and $G_{\gamma 5,7,9,10}$ proteins could combine to form a dimer, there would be 16 potential arrangements in PCa cells. Emerging evidences suggest that most pairs can indeed form, with some noted exceptions in specific expression systems $[4,9,25]$. For instance, $G_{\beta 1}$ can combine with $G_{\gamma 2}$ and $G_{\gamma 5}$ but not $G_{\gamma 3}$; and $G_{\beta 2}$ can form a pair with $G_{\gamma 5}$ but not with $G_{\gamma 1}$ [26]. Also, $G_{\beta 3}$ pairing with $G_{\gamma 1}$ and $G_{\gamma 2}$ is structurally impossible [9]. $G_{\gamma 13}$ can form stable dimers with $G_{\beta 1}, G_{\beta 3}$, and $G_{\beta 4}$, while $G_{\gamma 10}$ is capable of interacting with $G_{\beta 1}, G_{\beta 2}$, but not $G_{\beta 3}[9,27,28]$. Future $\mathrm{X}$-ray crystallography studies will be necessary to unravel the precise structural and functional relationship(s) among $\mathrm{G}$ protein subunit isoforms.

Malignant cells, which express a wide repertoire of chemokine receptors, respond to chemokines with increased directional migration, proliferation, and/or survival [29]. We have recently demonstrated CXCR5 expression in tissues obtained from $\mathrm{PCa}$ patients, and showed that elevated levels of CXCR5 correlate with advanced disease [15]. Furthermore, we established a role for CXCL13 and CXCR5 interaction in prostate tumor progression and elucidated some of the molecular and cellular processes mediated by activation of this chemokine receptor [14]. In confirmation we investigated the expression of CXCR5 and its association with $\mathrm{G}$ protein subunits in both androgen sensitive and hormone refractory PCa cells. However, five minutes after CXCL13 stimulation, the $G$ protein subunits $\left(G_{\alpha i 2}\right.$ and $\left.G_{\alpha q / 11}\right)$ that bind to CXCR5 were not detected in cell lysates. The plausible explanation for this finding is that binding of CXCL13 to CXCR5 causes conformational changes that elicit the classical dissociation of these $G$ proteins, allowing them to stimulate downstream signaling cascades. Indeed, static and dynamic light scattering measurements of protein complexes will be used to quantify the strength of these interactions, including potential homo- and hetero-associations. In addition to the stoichiometry of these protein-protein associations, future studies will also include isothermal titration calorimetry characterization of these interactions to provide information on the enthalpy, entropy and binding kinetics between these proteins.

Oncogenic mutations of $G_{\alpha i 2}$ protein have been identified in ovarian and adrenocortical tumors suggesting a potential role in cellular transformation [30]. $G_{\alpha i 2}$ has also been reported to promote B lymphocyte trafficking and motility within lymph nodes in response to CXCL13 [31]. The characteristic $G_{\alpha i 2}$ coupling to CXCR5, a chemokine receptor aberrantly expressed by $\mathrm{C} 4-2 \mathrm{~B}$ and $\mathrm{PC} 3$ cell lines, offers a new perspective on the role of $\mathrm{G}$ proteins in CXCL13:CXCR5-mediated PCa cell migration.

While the $\mathrm{LNCaP}$ cell line is androgen-responsive, C42B and PC3 cell lines have hormone-refractory properties
[20,32]. This might explain the differential expression of $\mathrm{G}$ proteins we observed in $\mathrm{LNCaP}$ and $\mathrm{C} 4-2 \mathrm{~B}$ cell lines, even though the $\mathrm{C} 4-2 \mathrm{~B}$ cell line was derived from LNCaP cells. Androgen is known to regulate the cellular composition of the normal prostate and acts on a set of specific genes, which impact the protein repertoire of a cell [33]. This dissimilarity in PCa cell line sensitivity to androgen might account for the variation in $\mathrm{G}$ protein expression, and could ultimately mandate CXCR5-mediated $\mathrm{G}$ protein coupling in these cell types. Our results also suggest that androgen receptor (AR) activation and/ or inhibition may contribute to $G$ protein expression in PCa tumors. However, defining the contributions of AR in CXCR5 signaling will be the subject of a different study.

It has been demonstrated that $\mathrm{G}$ protein $\alpha$ subunits undergo post-translational lipidation, which increase their affinities for $\mathrm{G}$ protein $\beta$ and $\gamma$ subunits. These covalent modifications largely determine which $G$ protein $\alpha$ isoforms associate with specific $G$ protein $\beta \gamma$-complexes [34]. Inhibition of the $G_{\beta \gamma}$ subunits in general prevents PCa formation and growth in vivo [35]. It is worth noting that a polymorphism in the gene encoding $G_{\beta 3}$ subunit is associated with oncogenesis and risk of bone metastasis in patients with breast cancer, while the homozygous $G_{\beta 3}$ genotype conferred protection against disease progression [36]. Hence, the identification of $\mathrm{G}_{\beta 3 / \gamma 9}$ coupling to CXCR5 is of considerable interest and the functional relevance of this finding is a matter for future studies. It has also been noted that free $G_{\beta \gamma}$ complexes can effect other second messengers, e.g., phospholipase A2 and phospholipase C, or gating ion channels, e.g., G protein coupled inward rectifying potassium channels and L-type calcium channels. While this has not been observed following CXCR5 signaling, future studies will be needed to determine the potential signaling events induced by the $\mathrm{G}_{\beta 3-\gamma 9}$ complex following CXCR5 stimulation.

We also found that $G_{\alpha 13}$ protein associates with CXCR5 following CXCL13 stimulation. While multiple scenarios could exist to explain this result, $G_{\alpha 13}$ association with active CXCR5 could be the product of ligandmediated $G$ protein switching. It has been reported that $\mathrm{G}$ protein isoforms switch their coupling to receptors in response to ligand binding in a cAMP-dependent protein kinase (PKA) fashion to presumably initiate a new set of signaling cascades [37]. This phenomenon has been described in $\mathrm{CHO}$ cells, where the $\beta_{2}$-adrenergic receptor switches its coupling specificity from $G_{\alpha s}$ to $G_{\alpha i}$ in response to agonist binding [38].

Previously it has been shown that CXCR4 is widely expressed by $\mathrm{PCa}$ cell lines and migration and invasive potential of these cells were significantly impaired by anti-CXCR4 antibodies [39]. In our study, we found a 
constitutive coupling of CXCR4 to CXCR5 and a likely oligomerization with other GPCRs upon CXCR5 activation (Figure 5). This interaction can sequester $\mathrm{G}_{\alpha 13}$ and/ or associated receptors to apparently diminish their functions, e.g. adhesion. While co-immunoprecipitation is considered the gold standard for determining proteinprotein interactions of endogenous untagged proteins, futures studies will be needed to ascertain the affinity and confirmation of these interactions. Indeed, it will be important for potential molecular drug development efforts to determine the binding constants and the precise regions where CXCR5 (or CXCR4) and $\mathrm{G}_{\alpha q / 11}, \mathrm{G}_{\alpha \mathrm{\alpha} 2}$, $\mathrm{G}_{\alpha 13}, \mathrm{G}_{\beta 3}$ and $\mathrm{G}_{\gamma 9}$ proteins interact.

The ability of GPCRs to differentially couple to multiple classes of $G$ proteins $\left(G_{\alpha i}, G_{\alpha q / 11}, G_{\alpha 12 / 13}\right)$ has also been described for sphingosine-1-phosphate receptors, and the liver pancreastatin receptor $[40,41]$. While the possibility of CXCR5 switching from $G_{\alpha i}$ to $G_{\alpha 13}$ signaling pathways requires further investigation, the possibility of its occurrence presents a means for tumor cells to acquire new signaling machinery that could promote disease progression. Hence, it is more likely that CXCR5 binds $G_{\alpha 13}$ protein as a mechanism to sequester and prevent it from signaling, which would favor Rac $>>$ RhoA activation and cell migration. To explain, $G_{\alpha 12 / 13}$ family of $G$ proteins have been shown to stimulate RhoA activation and subsequent actin cytoskeletal rearrangements characterized by the formation of stress fibers for focal adhesion [42].

RhoA activation causes the formation of stress fibers and focal adhesions. Rac activation leads to lamellipodia formation and membrane ruffling, while cdc42 activation results in filopodia formation. These cellular processes are particularly important for cell migration and adhesion [43]. Compelling evidence suggest that Rac are primarily activated by $G_{\alpha i}$ and $G_{\alpha q}$ subunits [44]. RhoA has shown to be activated downstream of $G_{\alpha 12 / 13}$ subunits and to a lesser extent by $G_{\alpha q}$, while $G_{\beta \gamma}$ complexes are thought to contribute to activation of both RhoA and Rac pathways through direct stimulation of PI3K [45].

\section{Conclusions}

We show differential G protein expression by PCa cell lines and establish specific heterotrimeric coupling to CXCR5 in an androgen-sensitive (LNCaP) and hormone refractory (C4-2B and $\mathrm{PC} 3$ ) manner. We also provide evidence for $G_{\alpha 13}$ protein association with CXCR5 following CXCL13 stimulation, which could inhibit or potentiate various cellular processes. Moreover, we identify for the first time the constitutive coupling of CXCR4 to

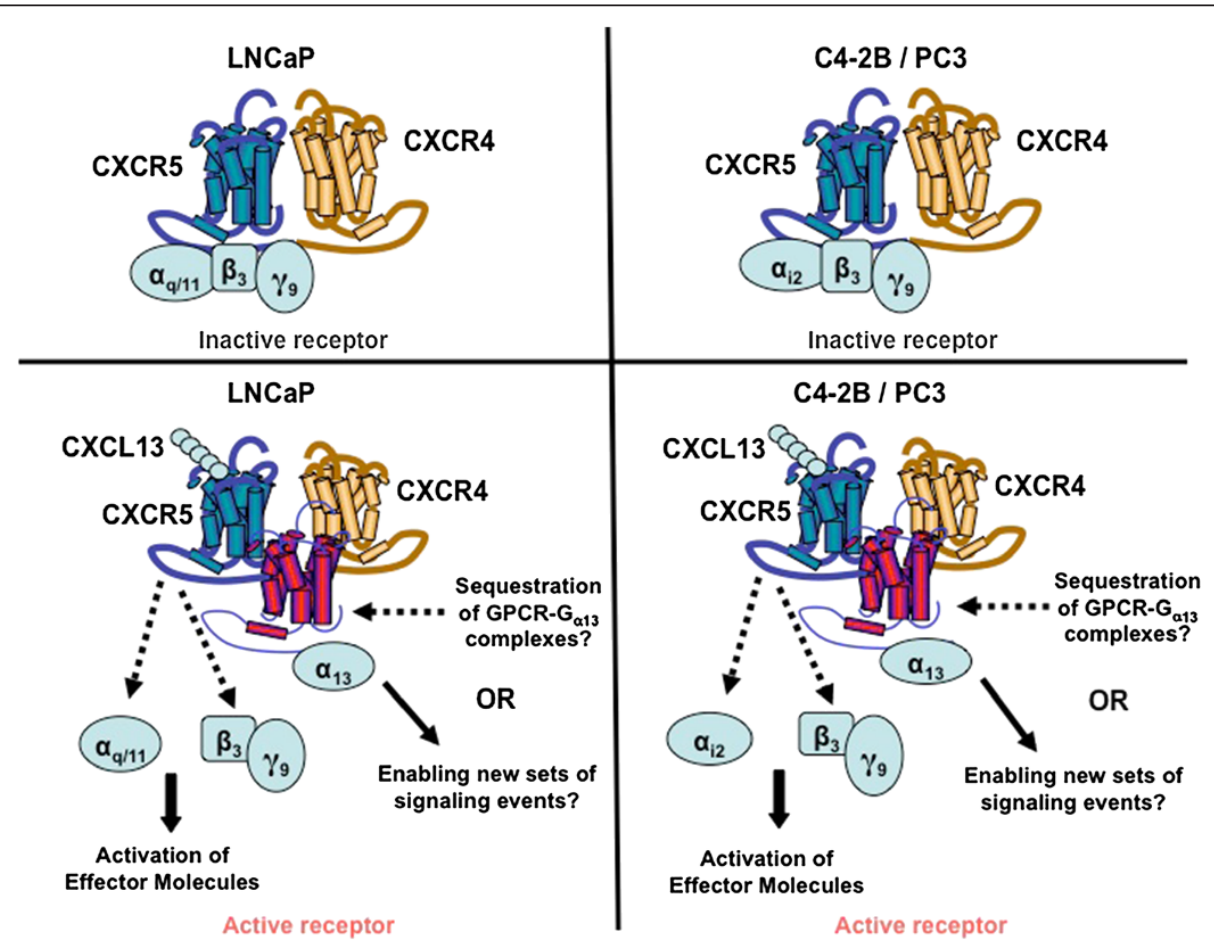

Figure 5 Hypothetical model of CXCR5 interactions in PCa cells. CXCR5 associates with CXCR4 and couples with $G_{a q / 11 /} G_{\beta 3}, G_{\gamma 9}$ heterotrimers in androgen-dependent $L N C a P$ cell line or $G_{a i 2} G_{33} G_{\gamma 9}$ heterotrimers in hormone refractory C4-2B and PC3 cell lines in the absence of its specific ligand, CXCL13. Upon CXCL13 stimulation, G proteins dissociate from CXCR5 to activate effector molecules. In addition, CXCL13-activated CXCR5 associates or sequesters $\mathrm{G}_{\mathrm{a} 13}$ protein favoring signals that would promote $\mathrm{PCa}$ cell motility. 
CXCR5. Clearly, there is much to learn about how specific heterotrimeric $\mathrm{G}$ protein compositions are regulated, and how these associations dictate unique signaling pathways. It will also be important to determine the clinical relevance of the $G_{\alpha q / 11 /} G_{\beta 3 /} G_{\gamma 9}$ heterotrimer in early and $G_{\alpha i 2 /} G_{\beta 3 /} G_{\gamma 9}$ in advanced or hormone refractory PCa.

Several observations have described chemokine receptor oligomer formation resulting in unusual $G$ protein signaling [46]. The hetero-dimerization between CCR2 and CCR5 has been extensively explored and suggests a mechanism of differential receptor coupling to pertussis toxin-sensitive to -insensitive G proteins [47,48]. Evidence also supports the ability of CCR5 to interact with non-chemokine receptors including opioid receptors [49]. While CXCR4 is present in almost all invasive cancers, CXCR5 has been implicated in advanced stages of chronic myelogenous leukemia, head and neck cancers, colon, and prostate cancer $[1,12,29,50]$. There is growing evidence to suggest transactivation of chemokine receptors will result in signal amplification at the receptor level, providing a means for tumor cells to metastasize and grow $[21,46]$.

The signaling cascade following CXCL13-CXCR5 interactions is indeed complex. These signals support Rac activation and invasion in $\mathrm{G}_{\alpha \mathrm{q} / \mathrm{i} 2}$ protein dependent fashion. Further, CXCR5 associates with CXCR4 and following activation can sequester $G_{\alpha 13}$ and/or associated receptors to seemingly diminish their functions.

No doubt, CXCR5 and/or CXCL13 blockade and specific $\mathrm{G}$ protein inhibition might prove to be effective therapeutic strategies to disrupt CXCR5 (and possibly CXCR4) signaling to abrogate PCa cell metastasis.

\section{Methods}

\section{Cell lines and culture}

Human prostate cancer cell lines (LNCaP, C4-2B, and PC3) and the epithelial cell line RWPE-1 derived from normal prostate were used in this study. All the cell lines were obtained from ATCC. To authenticate the cell lines, we carried out short tandem repeats genotyping. RWPE-1 cell line (ATCC \# CRL-11609) is an established normal prostate epithelial cell line that was cultured in keratinocyte serum free media (K-SFM) supplemented with bovine pituitary extract $(0.05 \mathrm{mg} / \mathrm{ml})$ and epidermal growth factor $(5 \mathrm{ng} / \mathrm{ml})$ at $37^{\circ} \mathrm{C}$ in a humidified atmosphere with $5 \% \mathrm{CO}_{2}$. LNCaP cell line (ATCC \# CRL-1740) is derived from the left supraclavicular lymph node of a metastatic prostate adenocarcinoma patient and is responsive to 5-alpha-dihydrotestosterone. $\mathrm{C} 4$-2B cell line is derived from the $\mathrm{LNCaP}$ cell line; however, it is hormone refractory. The PC3 cell line (ATCC \# CRL-1435) was derived from a bone metastasis of a grade IV prostatic adenocarcinoma patient. All three PCa cell lines were cultured in complete RPMI 1640 media supplemented with $10 \%$ fetal bovine serum (FBS) and maintained in a cell culture incubator at $37^{\circ} \mathrm{C}$ in a humidified atmosphere with $5 \% \mathrm{CO}_{2}$. Cell lines were serum starved overnight prior to treatment with $100 \mathrm{ng} / \mathrm{ml}$ of CXCL13 (Pepro Tech, NJ, USA) or $1 \mathrm{U} / \mathrm{ml}$ of thrombin (Sigma, MO, USA).

\section{Immunoprecipitation}

RWPE-1, LNCaP, C4-2B and PC3 cells were lysed in a cell lysis buffer containing 1\% NP40, 1\% Triton X-100, $0.25 \%$ deoxycholate, $100 \mathrm{mM} \mathrm{NaCl}, 50 \mathrm{mM}$ Tris $-\mathrm{HCl}$, pH7.4, and protease and phosphatase inhibitors (Roche, IN, USA). The protein concentrations of whole cell lysates were determined by bicinchoninic acid (BCA) protein determination assay (Pierce, IL, USA). To determine selective $\mathrm{G}$ protein isoforms coupled to CXCR5, equal amounts $(100 \mu \mathrm{g})$ of LNCaP, C4-2B, and PC3 cell lysates were incubated with $1 \mu \mathrm{g}$ of mouse anti-CXCR5 (R\&D systems, MN, USA), mouse anti- $G_{\alpha i 2}$, rabbit anti- $G_{\alpha q / 11}$, or goat anti- $\mathrm{G}_{\alpha 13}$ antibodies (Santa Cruz, CA, USA) for $2 \mathrm{~h}$ at $4^{\circ} \mathrm{C}$. Immune complexes were collected by adding $20 \mu$ of Agarose A/G PLUS beads (Santa Cruz, CA, USA) overnight at $4^{\circ} \mathrm{C}$. Following incubation protein complexes were washed twice with lysis buffer by centrifugation at $10,000 \times g$ for $10 \mathrm{~min}$ at $4^{\circ} \mathrm{C}$ and released from the beads by boiling in sample buffer for $5 \mathrm{~min}$. The resultant immunoprecipitates were further analyzed by immunoblot analysis.

\section{Immunoblotting and antibodies}

Western blot analysis was conducted on immunoprecipitants generated as described above or directly on cell lysates containing $50 \mu \mathrm{g}$ of protein. Samples were denatured by boiling in Laemmli buffer for $5 \mathrm{~min}$, resolved by electrophoresis on 4-15\% gradient SDS-polyacrylamide gel as needed, and transferred to nitrocellulose membranes using a semi-dry transfer cell system (Bio-Rad, CA, USA). Membranes were blocked for $1 \mathrm{~h}$ at room temperature (RT) in 5\% non-fat milk in 1X TTBS (30 mM Tris-Base, $150 \mathrm{mM} \mathrm{NaCl}$, and $0.1 \%$ Tween 20), followed by washing with 1X TTBS. Primary antibodies against G proteins $\alpha_{i 1}$, $\alpha_{i 2}, \alpha_{i 3}, \alpha_{s}, \alpha_{\mathrm{q} / 11}, \alpha_{12}, \alpha_{13}, \alpha_{16}, \beta_{1}, \beta_{2}, \beta_{3}, \beta_{4}, \beta_{5}, \gamma_{1}, \gamma_{2}, \gamma_{3}, \gamma_{4}$, $\gamma_{5}, \gamma_{7}, \gamma_{9}, \gamma_{10}, \gamma_{13}$, CXCR5 (Santa Cruz, CA, USA), and CXCR4 (R\&D systems, MN, USA) were added to the membranes and incubated overnight at $4^{\circ} \mathrm{C}$ in $5 \%$ non-fat milk. Membranes were then washed and corresponding horseradish peroxidase (HRP)-conjugated secondary antibodies (Santa Cruz, CA, USA) were added for $1 \mathrm{~h}$ followed by additional washes. Immunoreactive proteins were visualized by a chemiluminescent detection reagent (Amersham, PA, USA) on autoradiographic films. The blots were re-probed each time to stain different $G$ protein subunit isoforms. Following development for $\mathrm{G}$ proteins, 
all membranes were stripped and re-probed with antibody against GAPDH (Ambion, NY, USA) to ensure equal loading.

\section{Invasion assay}

PCa cell invasion was assessed using BD Matrigel ${ }^{\mathrm{Tm}}$ invasion chamber (BD Biosciences). Briefly, Matrigel inserts were hydrated for $2 \mathrm{~h}$ with $500 \mu \mathrm{l}$ of DMEM at $37^{\circ} \mathrm{C}$ with $5 \% \mathrm{CO}_{2}$. CXCL13 $(100 \mathrm{ng} / \mathrm{ml})$ or thrombin $(1 \mathrm{U} / \mathrm{ml})$ was added to the bottom chamber containing serum-free RPMI medium. LNCaP, C4-2B, and PC3 cells were transfected with $1 \mu \mathrm{g}$ control siRNA, $\mathrm{G}_{\alpha \mathrm{\alpha q} / 2}$ siRNA, or $G_{\alpha 13}$ siRNA duplex (Santa Cruz, CA, USA) prior to harvest, and added to the top chambers in serum-free RPMI medium at 10,000 cells per well. The cells were allowed to invade for $8 \mathrm{~h}$ at $37^{\circ} \mathrm{C}$ with $5 \%$ $\mathrm{CO}_{2}$. Non-invading cells on the upper surface of the membrane were removed with a cotton swab. The cells that migrated to the lower surface of the membrane were fixed with methanol at RT for $5 \mathrm{~min}$, stained with crystal violet for $2 \mathrm{~min}$, and washed with distilled water. The membranes were peeled and mounted on glass slides. Cells were then counted by microscopy at $40 \mathrm{X}$ magnification. Experiments were performed in triplicate and repeated three times.

\section{Rac and RhoA G-LISA activation assays}

Rac and RhoA activity were determined from cell lysates collected from LNCaP, C4-2B, and PC3 cells treated with or without CXCL13, thrombin, control siRNA, $G_{\alpha q / i 2}$ siRNA and/or $G_{\alpha 13}$ siRNA. PCa cells were transfected with $1 \mu \mathrm{g}$ of control, $G_{\alpha q / i 2}$ siRNA, or $G_{\alpha 13}$ siRNA duplexes (Santa Cruz, CA, USA) as before. Optimal knockdown of RNA and resulting protein knockdown occurred $72 \mathrm{~h}$ after transfection, which was confirmed by RT-PCR and Western blot analysis. Transfected PC3 cell cultures were pre-treated with media alone, $100 \mathrm{ng} / \mathrm{ml}$ of CXCL13 or $1 \mathrm{U} / \mathrm{ml}$ of thrombin for $30 \mathrm{~min}$. Subsequently, cultures were treated with these CXCR5 or PAR- 1 ligands to determine Rac and RhoA activities. After $10 \mathrm{~min}$. of stimulation, protein lysates were isolated and assayed using the colorimetric-based G-LISA ${ }^{\mathrm{mm}}$ Rac activity and luminescence-based G-LISA ${ }^{\mathrm{mm}}$ RhoA activation assay kits (Cytoskeleton, CO, USA), according to the manufacturer's instructions. Briefly, proteins were isolated using the provided cell lysis buffer and lysates were collected by centrifugation at $10,000 \mathrm{rpm}$ at $4^{\circ} \mathrm{C}$ for $2 \mathrm{~min}$. Protein concentrations from each sample were quantified and then adjusted to contain protein concentrations of $2 \mathrm{mg} / \mathrm{ml}$ for the assay. Absorbance and luminescence were detected as suggested by the manufacturer. Changes in Rac and RhoA activity among conditions are reported as fold difference normalized to the sample with no additions.

\section{Abbreviations}

ATCC: American Type Culture Collection; BCA: Bicinchoninic acid; GPCRs: G protein coupled receptors; FBS: Fetal bovine serum; HRP: Horseradish peroxidase; PCa: Prostate cancer; PI3K: Phosphoinositide-3 kinase; RPMI: Roswell Park Memorial Institute; SDS-PAGE: Sodium dodecyl sulfatepolyacrylamide gel electrophoresis; TTBS: Tris-Tween Buffered Saline.

\section{Competing interests}

The authors declare that they have no competing interests.

\section{Authors' contributions}

$\mathrm{CH}$ carried-out all experiments, quantified protein levels, and analyzed data with the assistance of PS, RS, PG, DT, and SS. JL conceived the study, participated in its design with all authors, coordinated and helped to draft the manuscript with the assistance of all authors. All authors read and approved the final manuscript.

\section{Acknowledgments}

The content of this manuscript benefited from many fruitful conversations with members of the Morehouse School of Medicine. This work benefited from the cooperation between investigators from the Morehouse School of Medicine and the Wallace Tumor Institute at the University of Alabama at Birmingham via the National Cancer Institute sponsored "Comprehensive Minority Institution / Cancer Center Partnership". This study was supported by funds from National Institute of Health Grants S21MD000101,

G12RR03034, G12MD007602, U54CA118638, and Department of Defense

(DoD) Prostate Cancer Research Program Award W81XWH-06-1-0562, and W81XWH-11-1-0109.

\section{Author details}

${ }^{1}$ Department of Pathology, Beth Israel Deaconess Medical Center, Harvard Medical School, Boston, MA, USA. ${ }^{2}$ School of Natural Sciences, Center of Life Sciences, Central University of Jharkhand, Brombe, Ranchi, India.

${ }^{3}$ Department of Microbiology, Biochemistry, \& Immunology, Morehouse School of Medicine, Atlanta, GA, USA. ${ }^{4}$ Laboratory of Immunology, National Institute on Aging, National Institute of Health, Bethesda, Maryland, USA.

Received: 12 February 2013 Accepted: 5 June 2013

Published: 18 June 2013

\section{References}

1. Boege FF, Neumann EE, Helmreich EJE: Structural heterogeneity of membrane receptors and GTP-binding proteins and its functional consequences for signal transduction. FEBS J 1991, 199:1-15.

2. Pierce $K L$, Premont RT, Lefkowitz RJ: Seven-transmembrane receptors. Nat Rev Mol Cell Biol 2002, 3:639-650.

3. Offermanns $S$, Simon Ml: Organization of transmembrane signalling by heterotrimeric G proteins. Cancer Surv 1996, 27:177-198.

4. Oldham WM, Hamm HE: Heterotrimeric G protein activation by G-proteincoupled receptors. Nat Rev Mol Cell Biol 2008, 9:60-71.

5. Beecroft MD, Taylor CW: Incremental Ca2+ mobilization by inositol trisphosphate receptors is unlikely to be mediated by their desensitization or regulation by luminal or cytosolic Ca2+. Biochem J 1997, 326:215-220.

6. Simon MIM, Strathmann MPM, Gautam NN: Diversity of G proteins in signal transduction. Science 1991, 252:802-808.

7. Downes GB, Gautam N: The $G$ protein subunit gene families. Genomics 1999, 62:544-552.

8. Clapham DE, Neer EJ: New roles for G-protein beta gamma-dimers in transmembrane signalling. Nature 1993, 365:403-406.

9. Clapham DE, Neer EJ: G protein beta gamma subunits. Annu Rev Pharmacol Toxicol 1997, 37:167-203.

10. Offermanns S: G-proteins as transducers in transmembrane signalling. Prog Biophys Mol Biol 2003, 83:101-130.

11. Spiegel AM: Defects in $G$ protein-coupled signal transduction in human disease. Annu Rev Physiol 1996, 58:143-170.

12. Waugh DJJ, Wilson C, Seaton A, Maxwell PJ: Multi-faceted roles for CXC-chemokines in prostate cancer progression. Front Biosci 2008, 13:4595-4604. 
13. Bonfil RD, Chinni S, Fridman R, Kim H-R, Cher ML: Proteases, growth factors, chemokines, and the microenvironment in prostate cancer bone metastasis. Urol Oncol 2007, 25:407-411.

14. Singh S, Singh R, Sharma PK, Singh UP, Rai SN, Chung LWK, Cooper CR, Novakovic KR, Grizzle WE, Lillard JW: Serum CXCL13 positively correlates with prostatic disease, prostate-specific antigen and mediates prostate cancer cell invasion, integrin clustering and cell adhesion. Cancer Lett 2009, 283:29-35.

15. Singh $S$, Singh $R$, Singh UP, Rai SN, Novakovic KR, Chung LWK, Didier PJ, Grizzle WE, Lillard JW: Clinical and biological significance of CXCR5 expressed by prostate cancer specimens and cell lines. Int J Cancer 2009, 125:2288-2295.

16. Amatruda TT, Steele DA, Slepak VZ, Simon MI: G alpha 16, a G protein alpha subunit specifically expressed in hematopoietic cells. Proc Natl Acad Sci USA 1991, 88:5587-5591.

17. Rodríguez-Frade JM, Martínez-A C, Mellado M: Chemokine signaling defines novel targets for therapeutic intervention. Mini Rev Med Chem 2005, 5:781-789.

18. Nguyen Q-D, Faivre S, Bruyneel E, Rivat C, Seto M, Endo T, Mareel M, Emami S, Gespach C: RhoA- and RhoD-dependent regulatory switch of Galpha subunit signaling by PAR-1 receptors in cellular invasion. FASEB J 2002, 16:565-576.

19. Tan W, Martin D, Gutkind JS: The Galpha13-Rho signaling axis is required for SDF-1-induced migration through CXCR4. J Biol Chem 2006, 281:39542-39549.

20. George SR, O'Dowd BF, Lee SP: G-protein-coupled receptor oligomerization and its potential for drug discovery. Nat Rev Drug Discov 2002, 1:808-820.

21. Rodríguez-Frade JM, Mellado M, Martínez-A C: Chemokine receptor dimerization: two are better than one. Trends Immunol 2001, 22:612-617.

22. García-Fernández MO, Solano RM, Sánchez-Chapado M, Ruiz-Villaespesa A, Prieto JC, Carmena MJ: Low expression of Galpha protein subunits in human prostate cancer. J Urol 2001, 166:2512-2517

23. Kelly P, Casey PJ, Meigs TE: Biologic functions of the G12 subfamily of heterotrimeric g proteins: growth, migration, and metastasis. Biochemistry 2007, 46:6677-6687.

24. Kelly P, Stemmle LN, Madden JF, Fields TA, Daaka Y, Casey PJ: A role for the G12 family of heterotrimeric $\mathrm{G}$ proteins in prostate cancer invasion. J Biol Chem 2006, 281:26483-26490.

25. Yan K, Kalyanaraman V, Gautam N: Differential ability to form the G protein betagamma complex among members of the beta and gamma subunit families. J Biol Chem 1996, 271:7141-7146.

26. Wu D, Katz A, Simon Ml: Activation of phospholipase $C$ beta 2 by the alpha and beta gamma subunits of trimeric GTP-binding protein. Proc Natl Acad Sci USA 1993, 90:5297-5301.

27. Blake BL, Wing MR, Zhou JY, Lei Q, Hillmann JR, Behe Cl, Morris RA, Harden TK, Bayliss DA, Miller RJ, Siderovski DP: G beta association and effector interaction selectivities of the divergent $\mathrm{G}$ gamma subunit $\mathrm{G}$ gamma (13). J Biol Chem 2001, 276:49267-49274.

28. Ray K, Kunsch C, Bonner LM, Robishaw JD: Isolation of CDNA clones encoding eight different human $G$ protein gamma subunits, including three novel forms designated the gamma 4, gamma 10, and gamma 11 subunits. J Biol Chem 1995, 270:21765-21771.

29. Zlotnik A: Chemokines and cancer. Int J Cancer 2006, 119:2026-2029.

30. Farfel Z, Bourne HR, liri $\mathrm{T}$ : The expanding spectrum of $\mathrm{G}$ protein diseases. N Engl J Med 1999, 340:1012-1020.

31. Han S-B, Moratz C, Huang N-N, Kelsall B, Cho H, Shi C-S, Schwartz O, Kehrl $\mathrm{JH}$ : Rgs1 and Gnai2 regulate the entrance of B lymphocytes into lymph nodes and B cell motility within lymph node follicles. Immunity 2005, 22:343-354.

32. Liu AY, Brubaker KD, Goo YA, Quinn JE, Kral S, Sorensen CM, Vessella RL, Belldegrun AS, Hood LE: Lineage relationship between LNCaP and LNCaPderived prostate cancer cell lines. Prostate 2004, 60:98-108.

33. Debes JD, Tindall DJ: The role of androgens and the androgen receptor in prostate cancer. Cancer Lett 2002, 187:1-7.

34. Casey PJ: Protein lipidation in cell signaling. Science 1995, 268:221-225

35. Bookout AL, Finney AE, Guo R, Peppel K, Koch WJ, Daaka Y: Targeting Gbetagamma signaling to inhibit prostate tumor formation and growth J Biol Chem 2003, 278:37569-37573.

36. Clar H, Langsenlehner U, Krippl P, Renner W, Leithner A, Gruber G, Hofmann G, Yazdani-Biuki B, Langsenlehner T, Windhager R: A polymorphism in the
$\mathrm{G}$ protein beta3-subunit gene is associated with bone metastasis risk in breast cancer patients. Breast Cancer Res Treat 2008, 111:449-452.

37. Martin NP, Whalen EJ, Zamah MA, Pierce KL, Lefkowitz RJ: PKA-mediated phosphorylation of the beta1-adrenergic receptor promotes $\mathrm{Gs} / \mathrm{Gi}$ switching. Cell Signal 2004, 16:1397-1403.

38. Zamah AM, Delahunty M, Luttrell LM, Lefkowitz RJ: Protein kinase A-mediated phosphorylation of the beta 2-adrenergic receptor regulates its coupling to $\mathrm{Gs}$ and $\mathrm{Gi}$. Demonstration in a reconstituted system. J Biol Chem 2002, 277:31249-31256.

39. Taichman RS, Cooper C, Keller ET, Pienta KJ, Taichman NS, McCauley LK: Use of the stromal cell-derived factor-1/CXCR4 pathway in prostate cancer metastasis to bone. Cancer Res 2002, 62:1832-1837.

40. Kim E-S, Kim J-S, Kim SG, Hwang S, Lee CH, Moon A: Sphingosine 1-phosphate regulates matrix metalloproteinase-9 expression and breast cell invasion through S1P3-Gaq coupling. J Cell Sci 2011, 124:2220-2230.

41. Santos-Alvarez J, Sánchez-Margalet V: $G$ protein $G$ alpha $q / 11$ and $G$ alpha $i 1,2$ are activated by pancreastatin receptors in rat liver: studies with GTP-gamma 35S and azido-GTP-alpha-32P. J Cell Biochem 1999, 73:469-477.

42. Buhl AM, Johnson NL, Dhanasekaran N, Johnson GL: G alpha 12 and $\mathrm{G}$ alpha 13 stimulate Rho-dependent stress fiber formation and focal adhesion assembly. J Biol Chem 1995, 270:24631-24634.

43. Gratacap MP, Payrastre B, Nieswandt B, Offermanns S: Differential regulation of Rho and Rac through heterotrimeric G-proteins and cyclic nucleotides. J Biol Chem 2001, 276:47906-47913.

44. Booden MA, Siderovski DP, Der CJ: Leukemia-associated Rho guanine nucleotide exchange factor promotes $\mathrm{G}$ alpha q-coupled activation of RhoA. Mol Cell Biol 2002, 22:4053-4061.

45. Whitehead IP, Zohn IE, Der CJ: Rho GTPase-dependent transformation by G protein-coupled receptors. Oncogene 2001, 20:1547-1555.

46. Mellado M, Rodríguez-Frade JM, Vila-Coro AJ, Fernández S, Martín de Ana A, Jones DR, Torán JL, Martínez-A C: Chemokine receptor homo- or heterodimerization activates distinct signaling pathways. EMBO J 2001, 20:2497-2507

47. Rodríguez-Frade JM, del Real G, Serrano A, Hernanz-Falcón P, Soriano SF, Vila-Coro AJ, de Ana AM, Lucas P, Prieto I, Martínez-A C, Mellado M: Blocking HIV-1 infection via CCR5 and CXCR4 receptors by acting in trans on the CCR2 chemokine receptor. EMBO J 2004, 23:66-76.

48. Vàzquez-Salat N, Yuhki N, Beck T, O'Brien SJ, Murphy WJ: Gene conversion between mammalian CCR2 and CCR5 chemokine receptor genes: a potential mechanism for receptor dimerization. Genomics 2007 90:213-224.

49. Chen C, Li J, Bot G, Szabo I, Rogers TJ, Liu-Chen LY: Heterodimerization and cross-desensitization between the mu-opioid receptor and the chemokine CCR5 receptor. Eur J Pharmacol 2004, 483:175-186.

50. Meijer J, Zeelenberg IS, Sipos B, Roos E: The CXCR5 chemokine receptor is expressed by carcinoma cells and promotes growth of colon carcinoma in the liver. Cancer Res 2006, 66:9576-9582.

doi:10.1186/1476-4598-12-64

Cite this article as: El-Haibi et al:: Differential G protein subunit expression by prostate cancer cells and their interaction with CXCR5. Molecular Cancer 2013 12:64.

\section{Submit your next manuscript to BioMed Central and take full advantage of:}

- Convenient online submission

- Thorough peer review

- No space constraints or color figure charges

- Immediate publication on acceptance

- Inclusion in PubMed, CAS, Scopus and Google Scholar

- Research which is freely available for redistribution 